\title{
The PSE-3D instability analysis methodology for flows depending strongly on two and weakly on the third spatial dimension
}

\author{
P. Paredes ${ }^{1}$, V. Theofilis ${ }^{1}$, D. Rodríguez ${ }^{2}$ and J.A. Tendero ${ }^{1}$ \\ ${ }^{1}$ School of Aeronautics, Universidad Politécnica de Madrid, Pza. Cardenal Cisneros 3, E-28040 Madrid, Spain \\ ${ }^{2}$ Division of Engineering and Applied Science, California Institute of Technology, Pasadena CA, 91125 USA
}

\begin{abstract}
The present contribution discusses the development of a PSE-3D instability analysis algorithm, in which a matrix forming and storing approach is followed. Alternatively to the typically used in stability calculations spectral methods, new stable high-order finitedifference-based numerical schemes for spatial discretization ${ }^{1}$ are employed. Attention is paid to the issue of efficiency, which is critical for the success of the overall algorithm. To this end, use is made of a parallelizable sparse matrix linear algebra package which takes advantage of the sparsity offered by the finite-difference scheme and, as expected, is shown to perform substantially more efficiently than when spectral collocation methods are used. The building blocks of the algorithm have been implemented and extensively validated, focusing on classic PSE analysis of instability on the flow-plate boundary layer, temporal and spatial BiGlobal EVP solutions (the latter necessary for the initialization of the PSE-3D), as well as standard PSE in a cylindrical coordinates using the nonparallel Batchelor vortex basic flow model, such that comparisons between PSE and PSE-3D be possible; excellent agreement is shown in all aforementioned comparisons. Finally, the linear PSE-3D instability analysis is applied to a fully three-dimensional flow composed of a counter-rotating pair of nonparallel Batchelor vortices.
\end{abstract}

\section{Nomenclature}

Abbreviations

CGL Chebyshev-Gauss-Lobatto

DNS Direct Numerical Simulation

EVP EigenValue Problem

DRP Dispersion-Relation-Preserving Finite Differences

FD-q Stable high order Finite Differences

ODE Ordinary Differential Equation

OSE Orr-Sommerfeld Equation

PDE Partial Differential Equation

PSE Parabolized Stability Equations

PSE-3D Three Dimensional Parabolized Stability Equations

SBP Summation by-Parts

Latin Symbols

$(x, y, z) \quad$ Cartesian coordinates

$(x, r, \theta) \quad$ Polar coordinates

$t \quad$ Time

*Correspondence to: pedro.paredes@upm.es

${ }^{\dagger}$ Research Professor, Associate Fellow AIAA 
$(U, V, W)$ Basic flow velocity vector components both in cartesian $(x, y, z)$ and in polar $(x, r, \theta)$ coordinates

$\overline{\mathbf{q}} \quad$ Fluid variables vector

q Unsteady perturbation vector

$\hat{\mathbf{q}} \quad$ Vector of amplitude functions

Q Basic flow vector

$\bar{q} \quad$ Fluid variable

$q \quad$ Perturbation component

$\hat{q} \quad$ Perturbation component amplitude

$Q \quad$ Basic flow component

$m \quad$ Azimuthal wavenumber in polar coordinates

$N \quad$ Number of nodes

Greek Symbols

$\alpha, \beta, \omega \quad$ Eigenvalues

$\theta \quad$ Phase function exponent or azimuthal angle in polar coordinates

$\Theta \quad$ Phase function

Superscript and underscript

()$^{\dagger} \quad$ Complex conjugate

()$_{x} \quad$ Derivative in $\mathrm{x}$ direction

\section{Introduction}

Linear stability theory, in part motivated by research into laminar-turbulent flow transition, has occupied a substantial part of fluid mechanics research for over a century. Numerical expediency has confined the bulk of the efforts into analyzing one-dimensional shear flows. The classic linear stability theory of Tollmien ${ }^{2}$ is mainly concerned with individual sinusoidal waves propagating in the boundary layer parallel to the wall. In this context, the parallel flow approximation is assumed, resulting in an ODE-based eigenvalue problem, which may be expressed as the Orr-Sommerfeld and Squire equations. ${ }^{3-5}$ The prediction of boundary layer transition based on solutions of the Orr-Sommerfeld equation (OSE) remains the only theoretically-founded approach in use in industry presently. However, discrepancies exist with experimental results, especially at the tip of the neutral curve of Blasius flow, particularly at low Reynolds numbers, as well as in boundary-layer flows in the presence of pressure gradients. The origin of the discrepancies was traced back to the existence of a small but not negligible wall-normal basic flow velocity component. An instability analysis concept of the multiple-scales class, which relaxes the parallel flow approximation was introduced in the 1990s of last century ${ }^{6-8}$ denominated Parabolized Stability Equations (PSE), owing to the change in the mathematical nature that the multiple-scales approach introduces to the linearized stability equations. A key advantage of the PSE over the OSE types of approaches is that it permits natural introduction of nonlinear mode interaction and has been shown to be in excellent agreement with spatial DNS both on flat-plate boundary layer ${ }^{7}$ and vortical flows. ${ }^{9}$ In-depth studies of the classic PSE have appeared in the literature in the course of the past two decades; ${ }^{7}, 10-13$ see Herbert ${ }^{14}$ for a review.

In a manner conceptually analogous to the Orr-Sommerfeld equation, global linear stability theory may consider basic flows which are inhomogeneous in two (rather than one) spatial directions. The amplitude functions of the associated small-amplitude perturbations are now inhomogeneous in the same two spatial directions upon which the basic flow depends and are periodic along the third. Three decades of research into this type of global instability analysis has also produced a wealth of information; see Theofilis ${ }^{15,16}$ for a review. However, it is recently becoming clear that the assumption of homogeneity in one spatial direction may be overtly restrictive even in as simple a flow as a single spatially-developing viscous vortex as seen in the results of Broadhurst et al., ${ }^{17}$ who analyzed a spatially-developing Batchelor vortex both by BiGlobal theory and DNS. In the first case in which the axial direction was artificially kept homogeneous, these authors predicted linear instability followed by nonlinear saturation. By contrast, the nonlinear development of instability in the Batchelor vortex is qualitatively different if the axial direction is kept homogeneous or if the vortex is allowed to evolve. Broadhurst and Sherwin ${ }^{18}$ found that if spiral-type instability is allowed 
to develop in an axially unconstrained manner, a streamwise loss in energy (initiated by the linear mode of instability), will lead to the formation of an internal stagnation point and ultimate vortex breakdown.

These findings motivate an analysis technique that can account for two inhomogeneous spatial directions alongside milder velocity and pressure gradients along the third, axial in a vortex flow, direction. This concept, denominated PSE-3D, is the main topic of the present work. After an overview of the available linear and nonlinear instability analysis methodologies in section II, the equations governing PSE-3D are presented in incompressible flow, for brevity, and discussed in some depth. The temporal and spatial BiGlobal eigenvalue problem is also presented in the same section; solutions to the latter problem are essential as initial conditions for the PSE-3D algorithm. Owing to the size of the associated numerical problems, attention has been paid to the efficient solution of the PDE-based eigenvalue problem; section III introduces high-order finite-difference schemes ${ }^{1}$ as alternatives to spectral collocation spatial discretization and compares results obtained. In the same section a sparse linear algebra methodology is introduced for the inversion of the matrices (which are formed and stored). Although the package utilized is parallelizable, only comparisons of serial solutions are shown. Section IV presents several validations of the building blocks of the newlydeveloped algorithms: solutions of both the temporal and spatial BiGlobal EVP are presented for two different flow geometries. In addition, solutions of the standard PSE problem, to which the PSE-3D may be reduced, have been obtained. In all cases excellent agreement is shown with available literature results. Finally, in Section V PSE-3D results obtained in an isolated and a counter-rotating pair of realistic, spatiallydiffusing vortices are presented for the first time. Concluding remarks are offered in Section VI.

\section{Theoretical analysis concepts}

\section{A. Modal Linear Stability Theory}

Hydrodynamic instability studies the behavior of a laminar flow field upon the introduction of smallamplitude perturbations, in order to improve the understanding of the processes involved in the onset of unsteadiness in low-Reynolds-number flows and the transition of laminar flow to a turbulent regime.

Three-dimensional Navier-Stokes equations of a viscous, incompressible fluid in dimensionless form and Cartesian coordinates are

$$
\begin{aligned}
\nabla \cdot \overline{\mathbf{u}} & =0 \\
\frac{\partial \overline{\mathbf{u}}}{\partial t}+\overline{\mathbf{u}} \cdot \nabla \overline{\mathbf{u}} & =-\nabla \bar{p}+\frac{1}{R e} \nabla^{2} \overline{\mathbf{u}}
\end{aligned}
$$

with the Reynolds number defined as:

$$
R e=U L / \nu
$$

being $U$ and $L$ the velocity and length scale respectively, and $\nu$ the kinematic viscosity.

The vector of fluid variables $\overline{\mathbf{q}}=(\overline{\mathbf{u}}, \bar{p})^{T}$ is decomposed into a steady mean flow $\mathbf{Q}$ and an unsteady small disturbance or perturbation $\mathbf{q}$ :

$$
\overline{\mathbf{q}}(\mathbf{x}, t)=\mathbf{Q}(\mathbf{x})+\mathbf{q}(\mathbf{x}, t)
$$

Table 1. Classification of linear stability theory concepts for analysis of a steady state $\overline{\mathrm{q}}$. The asterisk denotes a slowly-varying spatial direction. The dagger symbol denotes potential extension to include nonlinear mode interaction.

\begin{tabular}{lclll}
\hline Denomination & Assumptions & Basic State & Amplitude Functions & Phase Function $\Theta$ \\
\hline TriGlobal & - & $\mathbf{Q}(x, y, z)$ & $\hat{\mathbf{q}}(x, y, z)$ & $\exp \{-\omega t\}$ \\
PSE-3D $^{\dagger}$ & $\partial_{x} \mathbf{Q} \ll \partial_{y} \mathbf{Q}, \partial_{z} \mathbf{Q}$ & $\mathbf{Q}\left(x^{*}, y, z\right)$ & $\hat{\mathbf{q}}\left(x^{*}, y, z\right)$ & $\exp \left\{\int \alpha\left(x^{\prime}\right) d x^{\prime}-\omega t\right\}$ \\
BiGlobal & $\partial_{x} \mathbf{Q}=0$ & $\mathbf{Q}(y, z)$ & $\hat{\mathbf{q}}(y, z)$ & $\exp \{\alpha x-\omega t\}$ \\
PSE $^{\dagger}$ & $\partial_{x} \mathbf{Q} \ll \partial_{y} \mathbf{Q} ; \partial_{z} \mathbf{Q}=0$ & $\mathbf{Q}\left(x^{*}, y\right)$ & $\hat{\mathbf{q}}\left(x^{*}, y\right)$ & $\exp \left\{\int \alpha\left(x^{\prime}\right) d x^{\prime}+\beta z-\omega t\right\}$ \\
Local & $\partial_{x} \mathbf{Q}=\partial_{z} \mathbf{Q}=0$ & $\mathbf{Q}(y)$ & $\hat{\mathbf{q}}(y)$ & $\exp \{\alpha x+\beta z-\omega t\}$ \\
\hline
\end{tabular}


In linear stability theory, the perturbation term is usually written as the product of an amplitude function and a phase function, $\mathbf{q}=\hat{\mathbf{q}} \Theta$. Table 1 summarizes the different instability approaches arranged by increasing constrains to the basic flow.

Introducing this decomposition of the perturbed flow into the equations 2, and subtracting the basic flow (as it satisfies the Navier-Stokes and continuity equations itself) one arrives to the so-called perturbation equations

$$
\begin{aligned}
\nabla \cdot \mathbf{u} & =0 \\
\frac{\partial \mathbf{u}}{\partial t}+\mathbf{U} \cdot \nabla \mathbf{u}+\mathbf{u} \cdot \nabla \mathbf{U} & =-\nabla p+\frac{1}{R e} \nabla^{2} \mathbf{u}
\end{aligned}
$$

In these equations the $\mathbf{q}=\mathcal{O}(\varepsilon)$ perturbation terms, with $\varepsilon \ll 1$, have been retained, while the non-linear perturbation term $(\mathbf{u} \nabla \mathbf{u})$ is $\mathcal{O}\left(\varepsilon^{2}\right)$ and can be neglected. The linearization is correct while the disturbance amplitude remains small enough for the non-linear terms to be negligible. This statement should be kept in mind as the true non-linear system can become unstable under finite-amplitude disturbances, under conditions in which the linearized system will stay stable. One physical example of this is the laminarturbulent transition originated by the transient growth of initially small disturbances, that can be stable in a linear approach (see Butler ${ }^{19}$ for a review).

\section{B. PSE-3D}

In the most general case, the flowfield is three-dimensional and inhomogeneous in all three spatial directions. A numerical solution of the PDE-based eigenvalue problem resulting from the discretization of the three coupled direction, referred to a TriGlobal stability analysis, is possible but prohibitively expensive nowadays for most applications of interest. A parabolized variation of the three-dimensional stability equations (PSE3D) can be derived is the basic flow can be assumed to experience slow variations along one of the three spatial directions (see Table 1). In this manner, the three-dimensional eigenvalue problem is replaced by an initial value problem that is solved using a marching procedure along the slow direction.

\section{Formulation}

The derivation of the parabolized equations is based upon the introduction of a small parameter $\varepsilon=$ $O\left(R e^{-1}\right)$ and the slowly varying variable $\xi=\varepsilon x$. Then, the basic flow can be written in the form $\mathbf{Q}=[U, V, W, P]^{T}(\xi, y, z)$.

The quasi-parallel theory retains the Ansatz for BiGlobal modes as local solutions for every $\xi$ :

$$
\mathbf{q}(x, y, z, t)=\hat{\mathbf{q}}(x, y, z) \exp [i \theta(x, t)]
$$

with $\theta$ satisfying

$$
\theta(x, t)=\int_{x} \alpha(\xi) d \xi-\omega t
$$

Note that $\partial / \partial x=\varepsilon \partial / \partial \xi$ and therefore the above equations can be written with the differentiations respect to $x$ or $\xi$.

Within the PSE approximation, the streamwise derivatives of $\mathbf{q}$ take the form

$$
\frac{\partial^{k} \mathbf{q}}{\partial x^{k}}=i^{k}\left[\alpha^{k} \hat{\mathbf{q}}-i k \alpha^{k-1} \frac{\partial \hat{\mathbf{q}}}{\partial x}-i \frac{k(k-1)}{2} \alpha^{k-2} \frac{d \alpha}{d x} \hat{\mathbf{q}}\right] \exp [i \theta(x, t)],
$$

and for the first and second derivative, the above expression becomes:

$$
\begin{aligned}
\frac{\partial \mathbf{q}}{\partial x} & =\left(i \alpha \hat{\mathbf{q}}+\frac{\partial \hat{\mathbf{q}}}{\partial x}\right) \exp [i \theta(x, t)] \\
\frac{\partial^{2} \mathbf{q}}{\partial x^{2}} & =\left(-\alpha^{2} \hat{\mathbf{q}}+2 i \alpha \frac{\partial \hat{\mathbf{q}}}{\partial x}+i \frac{d \alpha}{d x} \hat{\mathbf{q}}\right) \exp [i \theta(x, t)]
\end{aligned}
$$

Therefore, by substituting (7) and the first and second streamwise derivatives $(10,11)$ into the linearized Navier-Stokes equations (6), and neglecting terms of order $\varepsilon^{2}$ as $\hat{\mathbf{q}}_{x x}, \alpha_{x x}$, products $\alpha_{x} \hat{\mathbf{q}}_{x}$, and their higher 
derivatives with respects to $x$, the incompressible linear PSE-3D equations are obtained:

$$
\begin{aligned}
\left(\mathcal{L}_{0}+U_{x}\right) \hat{u}+U_{y} \hat{v}+U_{z} \hat{w}+i \alpha \hat{p}+\left\{\left(U-\frac{2 i \alpha}{R e}\right) \hat{u}_{x}+\hat{p}_{x}\right\} & =\left[\frac{i}{R e} \frac{d \alpha}{d x} \hat{u}\right] \\
\left(\mathcal{L}_{0}+V_{y}\right) \hat{v}+V_{x} \hat{u}+V_{z} \hat{w}+\hat{p}_{y}+\left\{\left(U-\frac{2 i \alpha}{R e}\right) \hat{v}_{x}\right\} & =\left[\frac{i}{R e} \frac{d \alpha}{d x} \hat{v}\right] \\
\left(\mathcal{L}_{0}+W_{z}\right) \hat{w}+W_{x} \hat{u}+W_{y} \hat{v}+\hat{p}_{z}+\left\{\left(U-\frac{2 i \alpha}{R e}\right) \hat{w}_{x}\right\} & =\left[\frac{i}{R e} \frac{d \alpha}{d x} \hat{w}\right] \\
\hat{u}_{x}+i \alpha \hat{u}+\hat{v}_{y}+\hat{w}_{z} & =0
\end{aligned}
$$

where $\mathcal{L}_{0}=i \alpha U+V \mathcal{D}_{y}+W \mathcal{D}_{z}-\frac{1}{R e}\left(\mathcal{D}_{y y}+\mathcal{D}_{z z}-\alpha^{2}\right)-i \omega$.

Hence the PSE can be written in a compact form as:

$$
\mathcal{L} \hat{\mathbf{q}}+\mathcal{M} \frac{\partial \hat{\mathbf{q}}}{\partial x}=\left[\frac{d \alpha}{d x} \mathcal{N} \hat{\mathbf{q}}\right]
$$

where operators $\mathcal{L}, \mathcal{M}$ and $\mathcal{N}$ act only in $y$ and $z$. The term in square brackets can be neglected because $\mathcal{N}$ originates from the viscous terms and is of $\mathcal{O}\left(R e^{-1}\right)$ as is also the derivative $d \alpha / d x$.

An ambiguity exists in the PSE formulation, in which the changes in amplitude along the slow spatial direction can be contained both in the amplitude function $\hat{\mathbf{q}}$ or in the phase function of the Ansatz (8). A normalization condition is required in order to close the formulation of the problem (see Herbert ${ }^{11,14}$ for a review). In this work, the following normalization condition is used:

$$
\int_{\Omega} \hat{\mathbf{u}}^{\dagger} \frac{\partial \hat{\mathbf{u}}}{\partial x} d \Omega=\int_{\Omega} \frac{1}{2} \frac{\partial}{\partial x}|\hat{\mathbf{u}}|^{2} d \Omega=0
$$

where $\hat{\mathbf{u}}=(\hat{u}, \hat{v}, \hat{w})^{T}$. This normalization imposes that the kinetic energy of the shape functions remains independent of $x$. Thus, the amplitude growth is absorbed into the phase function.

\section{Numerical stability}

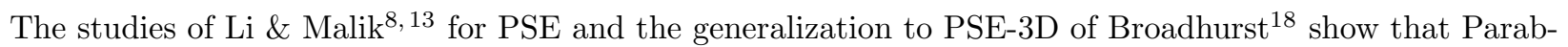
olized Stability Equations contain some residual ellipticity that makes them ill-posed. It can be shown ${ }^{13}$ that when using an implicit scheme along with sufficiently large streamwise steps, the upstream propagating modes are not captured in the numerical integration, thus permitting the stable integration of downstream moving instabilities. For a backward difference scheme in the marching direction, a limit of

$$
\Delta x>\frac{1}{|\alpha|}
$$

needs to be placed on the marching step size for stability purposes.

\section{BiGlobal Instability}

If the dependence in the third direction is neglected, the two-dimensional parallel flow is assumed and the BiGlobal instability can be used (see Theofilis ${ }^{15,16}$ for a review). This analysis will be necessary for the initialization of the PSE-3D marching integration and therefore is treated in this work.

\section{Formulation}

Assuming that the basic flow is only dependent on two out of the three spatial directions (see Table 1):

$$
\mathbf{Q}=[U, V, W, P]^{T}(y, z),
$$

the coefficients of the resulting eigenvalue problem are $x$-independent, and without loss of generality modal perturbations get the form

$$
\mathbf{q}(x, y, z, t)=\hat{\mathbf{q}}(y, z) \exp [i(\alpha x-\omega t)]
$$


The disturbances are still three-dimensional, but a sinusoidal dependence with the homogeneous $\mathrm{x}$ direction is assumed, with the periodicity length $L_{x}=2 \pi / \alpha$. Upon substitution of the ansatz 20 in the linearized equations 6 , results the system of equations

$$
\begin{aligned}
\mathcal{L} \hat{u}+U_{y} \hat{v}+U_{z} \hat{w}+i \alpha \hat{p} & =0 \\
\left(\mathcal{L}+V_{y}\right) \hat{v}+V_{z} \hat{w}+\hat{p}_{y} & =0 \\
\left(\mathcal{L}+W_{z}\right) \hat{w}+W_{y} \hat{v}+\hat{p}_{z} & =0 \\
i \alpha \hat{u}+\hat{v}_{y}+\hat{w}_{z} & =0
\end{aligned}
$$

where $\mathcal{L}=i \alpha U+V \mathcal{D}_{y}+W \mathcal{D}_{z}-\frac{1}{R e}\left(\mathcal{D}_{y y}+\mathcal{D}_{z z}-\alpha^{2}\right)-i \omega$.

The elliptic eigenvalue problem 21-24 must be complemented with adequate boundary conditions for the disturbance variables.

This equation can be written as a two-dimensional partial-derivative-based generalized eigenvalue problem that can be recast as the (linear) Temporal BiGlobal eigenvalue problem,

$$
\mathcal{A} \cdot \hat{\mathbf{q}}=\omega \mathcal{B} \cdot \hat{\mathbf{q}}
$$

where $\hat{\mathbf{q}}=[\hat{u}, \hat{v}, \hat{w}, \hat{p}]^{T}$ and the (nonlinear) Spatial BiGlobal eigenvalue problem,

$$
\mathcal{A} \cdot \hat{\mathbf{q}}=\sum_{k=1}^{2} \alpha^{k} \mathcal{B}_{k} \cdot \hat{\mathbf{q}} .
$$

The latter problem can be converted into a (larger by a factor equal to the degree of nonlinearity) linear eigenvalue problem, as in $\mathrm{Heeg}^{20}$, using the companion matrix method ${ }^{21,22}$, in which an auxiliary vector $\hat{\mathbf{q}}^{*}=[\hat{u}, \hat{v}, \hat{w}, \hat{p}, \beta \hat{u}, \beta \hat{v}, \beta \hat{w}]^{T}$ is defined, resulting in

$$
\mathcal{A} \cdot \hat{\mathbf{q}}^{*}=\alpha \mathcal{B} \cdot \hat{\mathbf{q}}^{*} .
$$

\section{Temporal BiGlobal instability Formulation}

Written using matrix notation, the operators $\mathcal{A}$ and $\mathcal{B}$ defining (25) become:

$$
\begin{aligned}
\mathcal{A} & =\left(\begin{array}{cccc}
\mathcal{L}_{T} & U_{y} & U_{z} & i \alpha \\
0 & \mathcal{L}_{T}+V_{y} & V_{z} & \mathcal{D}_{y} \\
0 & W_{y} & \mathcal{L}_{T}+W_{z} & \mathcal{D}_{z} \\
i \alpha & \mathcal{D}_{y} & \mathcal{D}_{z} & 0
\end{array}\right) \\
\mathcal{B} & =\left(\begin{array}{llll}
i & 0 & 0 & 0 \\
0 & i & 0 & 0 \\
0 & 0 & i & 0 \\
0 & 0 & 0 & 0
\end{array}\right)
\end{aligned}
$$

where $\mathcal{L}_{T}=i \alpha U+V \mathcal{D}_{y}+W \mathcal{D}_{z}-\frac{1}{R e}\left(\mathcal{D}_{y y}+\mathcal{D}_{z z}-\alpha^{2}\right)$. Here $\alpha \in \mathbb{R}$ is a wavenumber parameter, related with the periodicity length along the homogeneous spatial direction, $x$, through $\alpha=2 \pi / L_{x}$. The sought complex eigenvalue is $\omega=\left(\omega_{r}+i \omega_{i}\right)$, the real part being a circular frequency, while the imaginary part is the temporal amplification/damping rate.

\section{Spatial BiGlobal instability Formulation}

Written using matrix notation, the operators $\mathcal{A}$ and $\mathcal{B}$ defining (27) become:

$$
\mathcal{A}=\left(\begin{array}{ccccccc}
\mathcal{L}_{S} & U_{y} & U_{z} & 0 & 0 & 0 & 0 \\
0 & \mathcal{L}_{S}+V_{y} & V_{z} & \mathcal{D}_{y} & 0 & 0 & 0 \\
0 & W_{y} & \mathcal{L}_{S}+W_{z} & \mathcal{D}_{z} & 0 & 0 & 0 \\
0 & \mathcal{D}_{y} & \mathcal{D}_{z} & 0 & 0 & 0 & 0 \\
0 & 0 & 0 & 0 & \mathcal{I} & 0 & 0 \\
0 & 0 & 0 & 0 & 0 & \mathcal{I} & 0 \\
0 & 0 & 0 & 0 & 0 & 0 & \mathcal{I}
\end{array}\right)
$$




$$
\mathcal{B}=\left(\begin{array}{ccccccc}
-i U & 0 & 0 & -i & -\frac{1}{R e} & 0 & 0 \\
0 & -i U & 0 & 0 & 0 & -\frac{1}{R e} & 0 \\
0 & 0 & -i U & 0 & 0 & 0 & -\frac{1}{R e} \\
-i & 0 & 0 & 0 & 0 & 0 & 0 \\
\mathcal{I} & 0 & 0 & 0 & 0 & 0 & 0 \\
0 & \mathcal{I} & 0 & 0 & 0 & 0 & 0 \\
0 & 0 & \mathcal{I} & 0 & 0 & 0 & 0
\end{array}\right)
$$

where $\mathcal{L}_{T}=-i \omega+V \mathcal{D}_{y}+W \mathcal{D}_{z}-\frac{1}{R e}\left(\mathcal{D}_{y y}+\mathcal{D}_{z z}\right)$. Here $\omega \in \mathbb{R}$ is a real frequency parameter, while $\beta \in \mathbb{C}$ is the sought eigenvalue, the real part of which is related with the periodicity length along the homogeneous spatial direction, $z$, through $\alpha_{r}=2 \pi / L_{x}$ and the imaginary part, $\alpha_{i}$ is the spatial amplification/damping rate.

\section{PSE analysis with cylindrical-polar coordinates}

Since the base velocity profile for a trailing line vortex is axisymmetric, the general problem of the linearized stability can be studied by conventional PSE instability analysis, with different azimuthal wavenumber, instead using PSE-3D analysis. Therefore, this analysis can be utilized to validate the PSE-3D methodology.

With the same procedure as the incompressible linear PSE-3D, the incompressible linear PSE-2D equations are obtained in a cylindrical-polar coordinate framework.

Setting $(U, V, W)$ as the velocity components in the axial $x$, radial $r$ and azimuthal $\theta$ directions respectively, the basic flow is written in the form (see Table 1) $\mathbf{Q}=[U, \epsilon V, W, P]^{T}(\xi, r)$.

The perturbation and the phase function take the next form

$$
\mathbf{q}(x, r, \theta, t)=\hat{\mathbf{q}}(x, r) \exp [i \Theta(x, \theta, t)], \quad \Theta(x, \theta, t)=\int_{x} \alpha\left(x^{\prime}\right) d x^{\prime}+m \theta-\omega t,
$$

where $m \in \mathbb{Z}$ is the azimuthal wavenumber.

Finally, substituting in the linearized Navier-Stokes equations 6 in a cylindrical-polar coordinate frame, the axisymmetric PSE equations are obtained:

$$
\begin{aligned}
\mathcal{J} \hat{u}+U_{r} \hat{v}+i \alpha \hat{p}+U_{x} \hat{u}+\left\{\left(U-\frac{2 i \alpha}{R e}\right) \hat{u}_{x}+\hat{p}_{x}\right\} & =0 \\
\left(\mathcal{J}+\mathcal{K}+V_{r}\right) \hat{v}+\left(\frac{2 i m}{R e r^{2}}-\frac{2 W}{r}\right) \hat{w}+\hat{p}_{r}+\left\{\left(U-\frac{2 i \alpha}{R e}\right) \hat{v}_{x}\right\} & =0 \\
\left(W_{r}-\frac{2 i m}{R e r^{2}}+\frac{W}{r}\right) \hat{v}+\left(\mathcal{J}+\mathcal{K}+\frac{V}{r}\right) \hat{w}+\frac{i m}{r} \hat{p}+W_{x} \hat{u}+\left\{\left(U-\frac{2 i \alpha}{R e}\right) \hat{w}_{x}\right\} & =0 \\
i \alpha \hat{u}+\hat{u}_{x}+\frac{1}{r} \hat{v}+\hat{v}_{r}+\frac{i m}{r} \hat{w} & =0
\end{aligned}
$$

where $\mathcal{J}=i \alpha U+V \mathcal{D}_{r}+\frac{i m}{r} W-\frac{1}{R e}\left(\frac{1}{r}\left(\mathcal{D}_{r} r \mathcal{D}_{r}\right)-\frac{m^{2}}{r^{2}}-\alpha^{2}\right)-i \omega$ and $\mathcal{K}=\frac{1}{R e r^{2}}$.

\section{Spatial Local analysis in cylindrical-polar coordinates}

The local linear temporal instability of the swirling flows was originally studied by Khorrami et al. ${ }^{23}$ Later, Khorrami ${ }^{24,25}$ and Mayer \& Powell ${ }^{26}$ studied both the inviscid and viscous instability properties of an axisymmetric Batchelor vortex model flow in detail. The spatial local analysis is used in this work to obtain the initial condition for the PSE algorithm.

Assuming that the basic flow is only dependent on one out of the three spatial directions and with cylindrical-polar coordinates $[x, r, \theta]$ (see Table 1 ), it is written in the form $\mathbf{Q}=[U, V, W, P]^{T}(r)$.

The coefficients of the resulting eigenvalue problem are $(\mathrm{x}, \theta)$-independent, and without loss of generality modal perturbations get the form

$$
\mathbf{q}(x, r, \theta, t)=\hat{\mathbf{q}}(r) \exp [i(\alpha x+m \theta-\omega t)]
$$

where $m \in \mathbb{Z}$ is the azimuthal wavenumber. 
The disturbances are still three-dimensional, but a sinusoidal dependence with the homogeneous $(\mathrm{x}, \theta)$ directions is assumed, with the periodicity lengths $L_{x}=2 \pi / \alpha$ and $L_{\theta}=2 \pi / m$. Upon substitution of the ansatz 37 in the linearized equations 6 , results the system of equations

$$
\begin{aligned}
\mathcal{J}_{S} \hat{u}+U_{r} \hat{v}+i \alpha \hat{p} & =0 \\
\left(\mathcal{J}_{S}+\mathcal{K}+V_{r}\right) \hat{v}+\left(\frac{2 i m}{R e r^{2}}-\frac{2 W}{r}\right) \hat{w}+\hat{p}_{r} & =0 \\
\left(W_{r}-\frac{2 i m}{R e r^{2}}+\frac{W}{r}\right) \hat{v}+\left(\mathcal{J}_{S}+\mathcal{K}+\frac{V}{r}\right) \hat{w}+\frac{i m}{r} \hat{p} & =0 \\
i \alpha \hat{u}+\frac{1}{r} \hat{v}+\hat{v}_{r}+\frac{i m}{r} \hat{w} & =0
\end{aligned}
$$

where $\mathcal{J}_{S}=i \alpha U+V \mathcal{D}_{r}+\frac{i m}{r} W-\frac{1}{R e}\left(\frac{1}{r}\left(\mathcal{D}_{r} r \mathcal{D}_{r}\right)-\frac{m^{2}}{r^{2}}-\alpha^{2}\right)-i \omega$ and $\mathcal{K}=1 /\left(\operatorname{Re} r^{2}\right)$.

This equation can be written as an ordinary-differential-equation-based generalized eigenvalue problem that can be formally written as the temporal Local problem, with the same structure as 25 and the spatial Local problem as 27. Only the form of the spatial Local analysis in cylindrical-polar coordinates form is shown, since it is the one used in the subsequent analysis.

Written using matrix notation, the operators $\mathcal{A}$ and $\mathcal{B}$ defining (27) become:

$$
\mathcal{A}=\left(\begin{array}{ccccccc}
\mathcal{J}_{S} & U_{r} & 0 & i \alpha & 0 & 0 & 0 \\
0 & \mathcal{J}_{S}+\mathcal{K}+V_{r} & \frac{2 i m}{R e r^{2}}-\frac{2 W}{r} & \mathcal{D}_{r} & 0 & 0 & 0 \\
0 & W_{r}-\frac{2 i m}{R e r^{2}}+\frac{W}{r} & \mathcal{J}_{S}+\mathcal{K}+\frac{V}{r} & \frac{i m}{r} & 0 & 0 & 0 \\
0 & \frac{1}{r} \mathcal{D}_{r} r & \frac{i m}{r} & 0 & 0 & 0 & 0 \\
0 & 0 & 0 & 0 & \mathcal{I} & 0 & 0 \\
0 & 0 & 0 & 0 & 0 & \mathcal{I} & 0 \\
0 & 0 & 0 & 0 & 0 & 0 & \mathcal{I}
\end{array}\right)
$$

where $\mathcal{J}_{S}=U \mathcal{D}_{r}+\frac{i m}{r} V-\frac{1}{R e}\left(\frac{1}{r}\left(\mathcal{D}_{r} r \mathcal{D}_{r}\right)-\frac{m^{2}}{r^{2}}\right)-i \omega$.

The operator $\mathcal{B}$ follows the same structure than 31 .

\section{Boundary Conditions}

The elliptic eigenvalue problem 38-41 and the parabolic PSE system 33-34 must be complemented with adequate boundary conditions for the disturbance variables. For the main problem studied in this work, swirling flows, the boundary conditions, as originally derived by Batchelor ${ }^{27}$ are, in $r=0$ :

$$
\begin{array}{rll}
\frac{\partial \hat{u}}{\partial r}=\hat{v}=\hat{w}=\frac{\partial \hat{p}}{\partial r}=0 & \text { if } & m=0, \\
\hat{u}=\frac{\partial \hat{v}}{\partial r}=\hat{v}+m \hat{w}=\hat{p}=0 & \text { if } & |m|=1, \\
\hat{u}=\hat{v}=\hat{w}=\hat{p}=0 & \text { if } & |m|>1
\end{array}
$$

and that far from the wall all disturbances go to zero.

\section{Numerical considerations}

\section{A. Spatial discretization}

Numerical methods of formal high order of accuracy are necessary on analysis of vortical systems since the coupled spatial discretization of the eigenvalue problems 25 and 27 cannot be increased at will, due to memory limitations, in order for convergence to be achieved. The use of an analytic basic state eliminates concerns regarding the influence of insufficient resolutions for the basic state, and attention can be exclusively focused on the numerical solution of the eigenvalue problem. 
1. Spectral methods. These methods offer an optimal compromise between the highest accuracy possible and the necessity of reducing the amount of information to be stored. The reason of the high accuracy of (collocation) spectral methods lies on the use of high-order interpolating polynomials, comprising all the points in the discretization domain. Spectral methods use all the points and the error is $\varepsilon=\mathcal{O}\left((1 / N)^{N}\right) \rightarrow \mathcal{O}\left(e^{N}\right) .{ }^{28,29}$ Chebyshev-Gauss-Lobatto (CGL) points, indicated for the non periodic configurations of interest, are used here.

2. Stable high-order finite-difference methods based on non-uniform grid point distributions. These schemes were developed in order to reduce the ill-posedness of high-order finite-difference methods, that sometimes develop unphysical oscillations at the domain boundaries. For non-uniform grids, clustering of the discretization points close to the boundaries improves the stability of the resulting finite-difference operator. By following the philosophy behind the Chebyshev polynomials, a non-uniform grid for piecewise polynomial interpolations of degree $q \leq N$ is used, where $N+1$ is the total number of grid nodes. ${ }^{1}$ These schemes will be assigned by FD-q, where $q$ is the order of the scheme.

\section{Vortex instability analysis requirements}

The resolution requirements for the eigenvalue problem may be inferred from the structure of typical azimuthally inhomogeneous eigendisturbances of the Batchelor vortex. ${ }^{26}$ To select the domain, it is observed ${ }^{30}$ that the spatial structure of the eigenfunctions is confined within one-third of the radius at which the azimuthal velocity of the Batchelor vortex attains its maximum. This value is more than an order of magnitude smaller than the radius at which the basic flow itself decays to machine-zero level. A corollary of this observation is that vortex cores need substantially higher-density grids to be adequately resolved than the basic flow.

Asymptotic boundary conditions could be imposed in order to close the eigenvalue problem at the farfield, thus permitting a reduction in the domain extent. However, such boundary conditions would only be adequate to a particular problem (e.g. single vortex). For the sake of generality, Dirichlet boundary conditions are imposed to the disturbances. This results in the need of employing larger integration domains and appropriate mappings between CGL or FD-q grid $x \in[-1,1]$, what coincides with the standard grid of the finite differences used here, and the actual physical domain. The transformation used here for BiGlobal and PSE-3D analysis is (see Hein et al. ${ }^{30}$ ):

$$
\eta=\eta_{0}+\eta_{\infty} \frac{\tan \left(\frac{c \pi}{2} x\right)}{\tan \left(\frac{c \pi}{2}\right)}
$$

where $\eta$ is any the discretized spatial directions $x$ or $y$, while $\eta_{0}$ and $\eta_{\infty}$ are the respective center-point and far-field truncation locations.

In the Local and PSE-3D analysis, a different equation has to be used to mapped the calculation domain grid $r \in\left[0, r_{\infty}\right]$ into the CGL grid $x \in[-1,1]$. The transformation used in this context is:

$$
r_{j}=l \frac{1-x_{j}}{1+s+x_{j}}
$$

where $s=2 l / r_{\infty}$ and the length scale $l$ is defined as

$$
l=\frac{r_{\infty} r_{m}}{r_{\infty}-2 r_{m}}
$$

being $r_{\infty}$ the location where the calculation domain is truncated and $r_{m}$ the domain location that split in two half the number of collocation points.

\section{B. Eigenvalue computation}

The generalized eigenvalue problems must be constructed and solved employing adequate algorithms, taking into account the memory and CPU-time requirements when the matrices are formed and stored. Although the algorithm allows the use of dense or sparse linear algebra, the sparse version is much more efficient and it is the one used here. The complex matrices $\mathcal{A}$ and $\mathcal{B}$ are built using a modified version of the 
SPARSKIT2 library ${ }^{31}$ to work with complex arithmetic. To solve the eigenvalue problem, the Arnoldi algorithm ${ }^{32}$ is employed, combined with the MUMPS library ${ }^{33,34}$ (MUltifrontal Massively Parallel Solver, see http://graal.ens-lyon.fr/MUMPS/) to perform the LU-decomposition and solve the linear algebraic systems with the possibility of make serial and parallel computations.

The Arnoldi algorithm delivers a number of the eigenvalues in the neighborhood of a specified estimate (usually in the vicinity of the unstable/least-stable eigenvalue) at a small fraction of the cost pertinent to an equivalent QZ. The number of eigenvalues delivered is determined by the freely chosen Krylov subspace dimension, the limit of which is the leading dimension of matrices $\mathcal{A}$ and $\mathcal{B}$. A so-called shift-and-invert strategy is also implemented in our eigenvalue problem solver, according to which instead of 25 or 27 one solves

$$
\mathcal{C} \mathbf{X}=\mu \mathbf{X}, \quad \mathcal{C}=(\mathcal{A}-\sigma \mathcal{B})^{-1} \mathcal{B}, \quad \mu=\frac{1}{\omega-\sigma} .
$$

The Krylov subspace is constructed by

$$
\mathcal{K}_{m}=\operatorname{span}\left(\mathbf{v}, \mathcal{C} \cdot \mathbf{v}, \mathcal{C}^{2} \cdot \mathbf{v}, \ldots, \mathcal{C}^{m-1} \cdot \mathbf{v}\right)
$$

where $m$ is the dimension of the subspace. The vectors forming the basis are ordered by columns into the matrix $\mathbf{V}_{m}$. The projection of the linear operator on the basis $\mathbf{V}_{m}$ yields the relation

$$
\mathbf{V}_{m}^{H} \mathcal{C} \mathbf{V}_{m}=\mathbf{H}_{m}
$$

where $\mathbf{H}_{m}$ is an upper triangular Hessenberg matrix. The restriction of the eigenvalue problem to the Krylov subspace $\mathcal{K}_{m}$ is

$$
\mathbf{H}_{m} \mathbf{V}_{m}^{H} \cdot \hat{\mathbf{q}}=\mu \mathbf{V}_{m}^{H} \cdot \hat{\mathbf{q}} .
$$

The eigenvalues of the Hessenberg matrix $\mathbf{H}_{m}$ are approximations to the $m$ largest eigenvalues of the original problem. If $y_{i}^{(m)}$ is the eigenvector corresponding to the $i$-th eigenvalue of $\mathbf{H}_{m}$ the corresponding approximation to the eigenvector of the original problem, known as Ritz vector, is

$$
\hat{\mathbf{q}}_{i}=\mathbf{V}_{m} \cdot \mathbf{y}^{(m)} \text {. }
$$

\section{Solution procedure of the PSE-3D}

The streamwise derivative is approximated by the implicit backward Euler scheme:

$$
\left(\hat{\mathbf{q}}_{x}\right)_{j+1} \approx \frac{1}{\Delta x_{j}}\left(\hat{\mathbf{q}}_{j+1}-\hat{\mathbf{q}}_{j}\right)
$$

where $j \leq 0$ is the step index and $\hat{\mathbf{q}}_{j}=\hat{\mathbf{q}}_{j}(y, z)=\hat{\mathbf{q}}\left(x_{j}, y, z\right)$.

Equation 16 then assumes the form

$$
\left[\Delta x_{j} \mathcal{L}_{j+1}^{n}+\mathcal{M}_{j+1}^{n}\right] \hat{\mathbf{q}}_{j+1}^{n}=\mathcal{M}_{j+1}^{n} \hat{\mathbf{q}}_{j}
$$

where $n$ is the iteration index. Starting with an initial guess $\alpha_{j+1}^{0}=\alpha_{j}$, according to the first approximation $\alpha_{x}=0, \hat{\mathbf{q}}_{j+1}^{0}$ is obtained from (16). The normalization condition is used then to estimate a new $\alpha_{j+1}$ :

$$
\alpha_{j+1}^{n+1}=\alpha_{j+1}^{n}-\frac{i}{\Delta x_{j}} \frac{\int_{\Omega} \hat{\mathbf{u}}_{j+1}^{\dagger n}\left(\hat{\mathbf{u}}_{j+1}^{n}-\hat{\mathbf{u}}_{j}\right) d \Omega}{\int_{\Omega}\left|\hat{\mathbf{u}}_{j+1}^{n}\right|^{2} d \Omega}
$$

where an implicit backward Euler scheme has also been used for (17). Once the wavenumber is updated, Equation 55 is solved for the new shape functions, and the value of $\alpha$ can be reevaluated again. The iteration continues until the normalization condition is satisfied to within some specified residual $r$ (i.e. $\left.\left|\alpha^{n+1}-\alpha^{n}\right|<r\right)$.

\section{Validations}

\section{A. Temporal Local Analysis of Plane Poiseuille Flow}

In order to study the convergence of the finite difference scheme used for the spatial discretization, the temporal local stability analysis of the plane Poiseuille flow is considered. The Orr-Sommerfeld equation is 

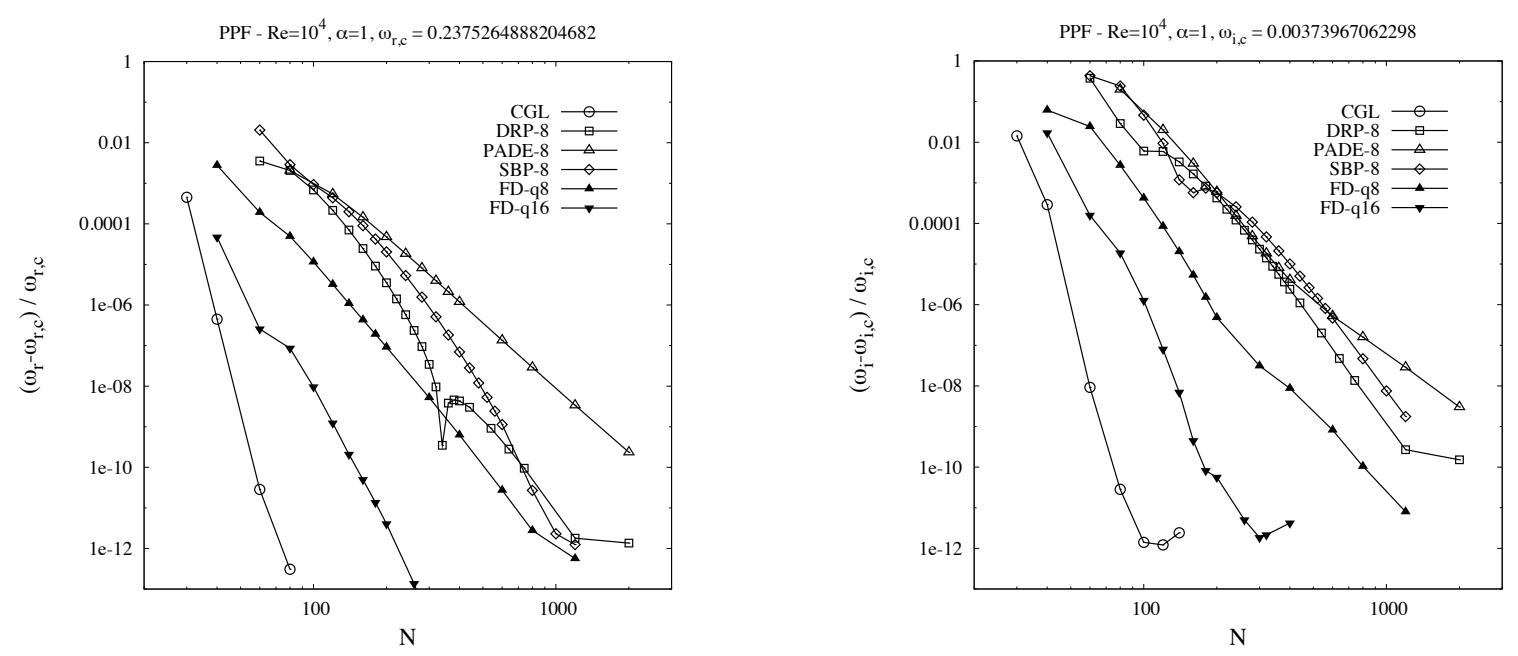

Figure 1. Convergence curves of real (left) and imaginary (right) parts of the most unstable mode of PPF at $R e=10000, \alpha=1$ and $\beta=0$ for different spatial schemes.

solved for $R e=10000$, streamwise wavenumber $\alpha=1$ and spanwise wavenumber $\beta=0$, for which the leading eigenvalue is $\omega_{r}+i \omega_{i}=0.2375264888204682+i 0.0037396706229799 .^{35}$ Figure 1 shows the convergence histories corresponding to different differentiation schemes: Padé ${ }^{36}$ (PADE), Dispersion-Relation-Preserving Finite Difference ${ }^{37}$ (DRP), Summation by-Parts operators for finite different approximations ${ }^{38,39}$ (SBP), all of them of $8^{\text {th }}$-order. $8^{\text {th }}$-order and $16^{\text {th }}$-order stable finite differences based on non-uniform grid point distributions $^{1}$ (FD-q8 and FD-q16, respectively), and Chebyshev-Gauss-Lobatto differentiation (CGL) are also used. All the $8^{\text {th }}$-order finite-difference schemes exhibit a similar behavior, with the FD-q8 attaining a better convergence that the others. As expected, the convergence rate for FD-q16 and CGL is better. However, the higher degree of sparsity in the $8^{\text {th }}$-order schemes makes them more efficient in terms of the numerical solution.

\section{B. Nonparallel instability of the Blasius boundary layer flow}

The boundary layer flow over a flat plate at zero pressure gradient is considered in order to validate the PSE implementation presented in this work. The PSE are made dimensionless using the far-field velocity $U_{0}$ and the displacement thickness at some arbitrary location $\delta_{0}=\delta_{r}\left(x_{0}\right)$. The Reynolds number is defined as $R e_{0}=U_{0} \delta_{0} / \nu$. In order to compare with classic results from linear stability theory, a new (local) Reynolds number is defined as $R e=\left(U_{0} x / \nu\right)^{1 / 2}$, and also a frequency parameter $F=\omega / R e_{0}$. The evolution of a two-dimensional Tollmien-Schlichting wave is considered for the validation, at $\omega=0.0344$ and $R e_{0}=400$. The initial condition is given by the solution of the spatial Orr-Sommerfeld equation at the inflow boundary. Figure (2,left) shows the imaginary part of the wavenumber evolution in $x$ compared with Herbert. ${ }^{11}$ Figure (2,right) compares the neutral stability curve obtained by Gaster ${ }^{40}$ using a multiple-scales approach, and the one delivered by the present PSE implementation.

\section{Results}

\section{A. Basic flow}

\section{The realistic Batchelor Vortex}

The model vortex proposed by Batchelor, ${ }^{27}$ as an asymptotic steady solution of the Navier-Stokes equations for $x \rightarrow \infty$ is used here as the basic flow. The free-stream velocity $U_{0}$ and reference core radius $\delta_{0}\left(x_{0}\right)$ at some arbitrary axial location are used for the non-dimensionalization. The Reynolds number is then defined as 

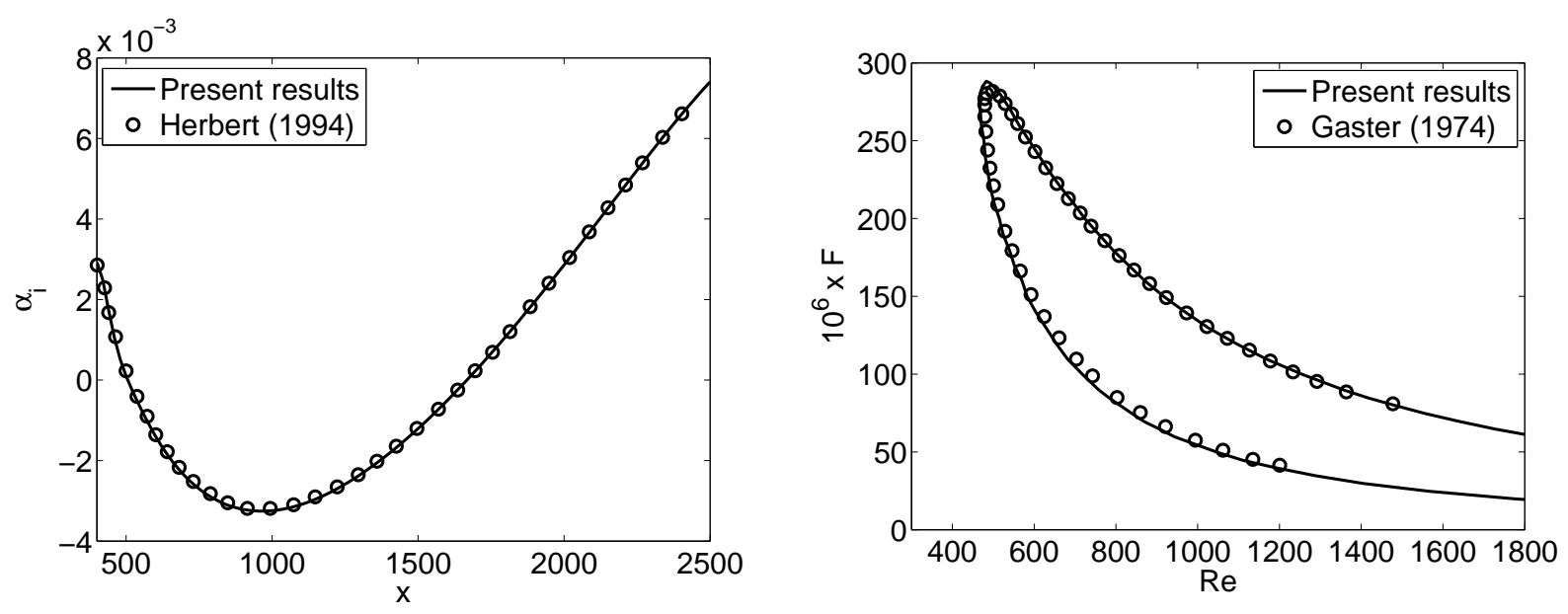

Figure 2. (Left) Evolution of the wavenumber $\alpha$ in a Blasius boundary layer at $R e_{0}=400, \omega=0.0344$ and $\beta=0$. (Right) Neutral curves for nonparallel Blasius flow according to PSE and Gaster (1974).

$$
R e_{0}=\frac{U_{0} \delta_{0}}{\nu}
$$

With $U_{x}, U_{r}$ and $U_{\theta}$ denoting the dimensionless velocity components in the axial $x$, radial $r$ and azimuthal $\theta$ directions, the model vortex is defined by ${ }^{41}$

$$
U_{x} \approx 1-\gamma(x) \exp \left(-r^{2} / \delta(x)^{2}\right), \quad U_{r} \approx 0, \quad U_{\theta} \approx \frac{\kappa}{r}\left(1-\exp \left(-r^{2} / \delta(x)^{2}\right)\right)
$$

where

$$
\kappa=\text { constant }, \quad \gamma(x)=\kappa^{2} \frac{R e_{0} \log \left(x / R e_{0}\right)}{8 x}, \quad \delta(x)=2 \sqrt{x / R e_{0}} .
$$

The quantity $\delta(x)$ corresponds physically to the dimensionless local vortex core radius, while $\gamma(x)$ is the axial velocity defect and $q$ is a swirl strength parameter.

While the asymptotic model assumes that the radial velocity vanishes, the continuity equation can be used in order to calculate a $U_{r}$ component:

$$
\left(\frac{1}{r} \frac{\partial}{\partial r} r\right) U_{r}=-\frac{\partial}{\partial x} U_{x}, \quad U(0)=0
$$

This correction is small compared to the dominant axial and azimuthal velocity components, but ensures that the basic flow will be divergence-free.

A Cartesian coordinate system is used in the solution of the BiGlobal eigenvalue problem and in the PSE-3D analysis. In this coordinate system, the plane orthogonal to the axial direction is defined by the coordinates $y$ and $z$, so that the radial direction becomes $r^{2}=\left(y-y_{n}\right)^{2}+\left(z-z_{n}\right)^{2}$. $\left(y_{n}, z_{n}\right)$ denotes the center of the $n$ vortex. In the new coordinate system, the three dimensional vortex flowfield is written as

$$
\begin{aligned}
U & =1-\gamma(x) \exp \left(\frac{-r^{2}}{\delta(x)^{2}}\right) \\
V & =-\frac{\kappa}{r^{2}}\left(z-z_{n}\right)\left(1-\exp \left(\frac{-r^{2}}{\delta(x)^{2}}\right)\right)+\frac{y-y_{n}}{r} U_{r} \\
W & =\frac{\kappa}{r^{2}}\left(y-y_{n}\right)\left(1-\exp \left(\frac{-r^{2}}{\delta(x)^{2}}\right)\right)+\frac{z-z_{n}}{r} U_{r}
\end{aligned}
$$




\section{The q-Vortex}

The q-Vortex flow proposed by Lessen ${ }^{42,43}$ is a particularization of the previous basic flow, in the case that the dependence of the flowfield on the axial direction is neglected. In this case, $\gamma$ and $\delta$ are constant, and the swirl parameter $q$ can be defined as

$$
q(x)=\frac{\kappa}{\gamma(x) \delta(x)} .
$$

The parallel-flow version of equations (58) is:

$$
U_{x}=a+\exp \left(-r^{2}\right), \quad U_{r}=0, \quad U_{\theta}=\frac{q}{r}\left\{1-\exp \left(-r^{2}\right)\right\}
$$

where $q=\frac{\Gamma}{2 \pi U_{c} \delta}$ is the swirl parameter, $a=U_{0} / U_{c}$, being $U_{c}$ the core vortex velocity, $U_{0}$ the free-stream velocity and $\delta$ the core radius. The Reynolds number is then defined by these velocity and length scales as

$$
R e=\frac{U_{c} \delta}{\nu}
$$

Note that the reference has been $U_{0}$ used previously has been replaced by the core velocity $U_{c}$. This is done on account that the simplification $a=0^{26}$ is used, equivalent to considering the reference system and the vortex moving with a constant axial velocity. In this manner, the real part of any eigenvalue found will give the disturbance velocity relative to the uniform axial velocity of the outer flow with the advantage of saving memory in the computations.

\section{B. BiGlobal Stability of the q-Vortex}

The stability of the parallel q-vortex flow is addressed here. While the q-vortex basic flow is axisymmetric and using the Orr-Sommerfeld equation written in cylindrical coordinates suffices to recover the modal stability of the flow, ${ }^{24,26}$ the more sophisticated BiGlobal stability analysis is used here. A reason for this is that the recovery of the results in the literature for the temporal stability of the flow serve as validation for the present implementation, as well as an useful test for the convergence of the results with the spatial discretization in the plane. In addition, the solution of the spatial stability problem provides an approximation to the initial conditions to be imposed in the PSE-3D solution.

\section{Temporal Analysis}

The temporal stability problem was solved for the parameters $q=0.475, R e=100, \alpha=0.418$, in order to compare with Mayer and Powell. ${ }^{26}$ The domain was truncated at $y_{\infty}=z_{\infty}=40$, where the variations in the basic flow are negligible. Three different discretization methods were tested: the spectral CGL with stretching parameter (see equation 46) $c=0.975$, and stable finite differences FD-q8 and FD-q10 with $c=0.93$. Table 2 shows the convergence of the leading eigenvalue with increasing resolutions for the different discretizations, and compares with Ref. 26. The converged eigenvalues agree reasonably well for the three differentiation methods, converging to a value with absolute difference lower than $10^{-7}$ for FD-q8 and $10^{-9}$ for FD-q10 and CGL. Note that the numerical solution using the highest resolution $\left(N=70^{2}\right)$ for CGL required 4935 Mbytes of memory, while in the case of FD-q10 $\left(N=100^{2}\right)$ the memory used was 1496 Mbytes, illustrating the performance improvement of the combination of high-order finite-difference schemes in conjunction with sparse storage and algebra.

\section{Spatial BiGlobal Analysis}

Spatial analyses were performed for the q-vortex at $R e=1200$ and $q=0.8$. Temporal stability results for the same case were presented by González et al. ${ }^{44}$ The spatial discretization of the problem used $N=100^{2}$ collocation points with the mapping parameter $c=0.965$, and FD-q12 for the numerical differentiation. Figure (3,left) shows the eigenspectrum at $R e=1200, q=0.8$ and $\omega=-2$. A branch of eigenvalues appear for $\alpha_{r}=0$ and $\alpha_{i}<0$, which corresponds to a discretized spectrum of upstream moving pressure waves. This branch is suppressed in the PSE approximation in order to permit a stable numerical integration along the axial direction, as discussed by Li and Malik. ${ }^{12}$ In addition, different branches of discrete eigenvalues are found, describing near-vertical lines. The discrete modes present a periodic structure in the azimuthal 
Table 2. Temporal BiGlobal instability analysis of the q-vortex flow at $q=0.475, R e=100$ and $\beta=0.418$.

\begin{tabular}{crll}
\hline $\begin{array}{c}\text { Spatial } \\
\text { scheme }\end{array}$ & $N^{2}$ & $\omega_{r} \times 10^{-2}$ & $\omega_{i} \times 10^{-3}$ \\
\hline MP & & & 9.61 \\
\hline CGL & $20^{2}$ & 2.833 & 9.64 \\
& $30^{2}$ & 2.8352 & 9.617 \\
& $40^{2}$ & 2.835189 & 9.616908 \\
& $50^{2}$ & 2.8351897 & 9.6169098 \\
& $60^{2}$ & 2.83518966 & 9.61690985 \\
& $70^{2}$ & 2.835189660 & 9.616909850 \\
\hline FD8 & $40^{2}$ & 2.835 & 9.62 \\
& $60^{2}$ & 2.8352 & 9.617 \\
& $80^{2}$ & 2.835183 & 9.6167 \\
& $100^{2}$ & 2.8351898 & 9.616912 \\
\hline FD10 & $40^{2}$ & 2.8353 & 9.617 \\
& $60^{2}$ & 2.8351899 & 9.61698 \\
& $80^{2}$ & 2.8351895 & 9.616913 \\
& $100^{2}$ & 2.83518965 & 9.61690986 \\
\hline
\end{tabular}

direction, with all the modes inside each branch having the same wavenumber $m$. Note that when a onedimensional stability analysis such as the Orr-Sommerfeld equation in cylindrical coordinates is used, the wavenumber $m$ is a parameter of the problem. The BiGlobal analysis recovers the results of several local analysis at the same time. The most unstable mode, pointed by an arrow in the spectrum is plotted in figure (3,right). Figure 4 shows the dependence of the of the discrete modes with the real frequency $\omega$. In the range of frequencies analyzed, the most unstable mode occurs for $m=4$, frequency $\omega \approx-0.8$ and $\alpha_{r} \approx-3$, in good agreement with the results of temporal analysis. ${ }^{44}$

\section{Stability analysis of a realistic isolated vortex}

The three-dimensional flowfield of a realistic isolated vortex is given by Equations 61, 62 and 63, with the parameter values $\gamma_{0}=0.9, \delta_{0}=1.0$ and $\kappa=-1.14$. The initial position is taken equal to the reference position for the Reynolds number, $x_{0}=R e_{0} / 4$. PSE in a cylindrical-polar coordinates frame and PSE-3D are employed as a cross-validation for both methodologies.

Initial conditions need to be specified for the PSE solution. The most common method used in conventional PSE is to use the local analysis of weakly nonparallel flow ${ }^{11}$ in order to obtain a wave number $\alpha$ and shape function $\hat{\mathbf{q}}$ at initial axial location for an specific $R e_{0}, \omega$. In this paper, the parallel-flow approximation along the axial direction is considered, and the spatial BiGlobal problem solved to obtain a first approximation to the wavenumber and shape functions. Afterwards, this result is refined by PSE iterations that introduce the non-parallel effects in the solution. Figure 5 show how the iterations using PSE-3D operator in the initial station alter the complex wavenumber. A small number of iterations is required for convergence.

Figure 6 compares the evolution of the complex wavenumber $\alpha$ along the axial direction obtained using the two different methodologies, namely the PSE in cylindrical coordinates and PSE-3D. In the case of the cylindrical coordinates, a CGL spatial discretization with $N_{r}=100$ and mapping parameter $r_{m}=3$ (see equation 47) was used. $N=120^{2}$ points with $c=0.96$ were used to discretize the $y_{\infty}=z_{\infty}=40$ domain, and FD-q10 were for the differentiation. Reasonably good agreement exists between the results of the two methodologies.

Figure 7 compares the three-dimensional solutions for the isolated vortex corresponding to a spatial BiGlobal analysis of the parallel q-vortex used at the initial axial location (upper plot), and the non-parallel PSE-3D solution (lower plot). The spread of the vortex downstream, caused by viscous diffusion, reduces 

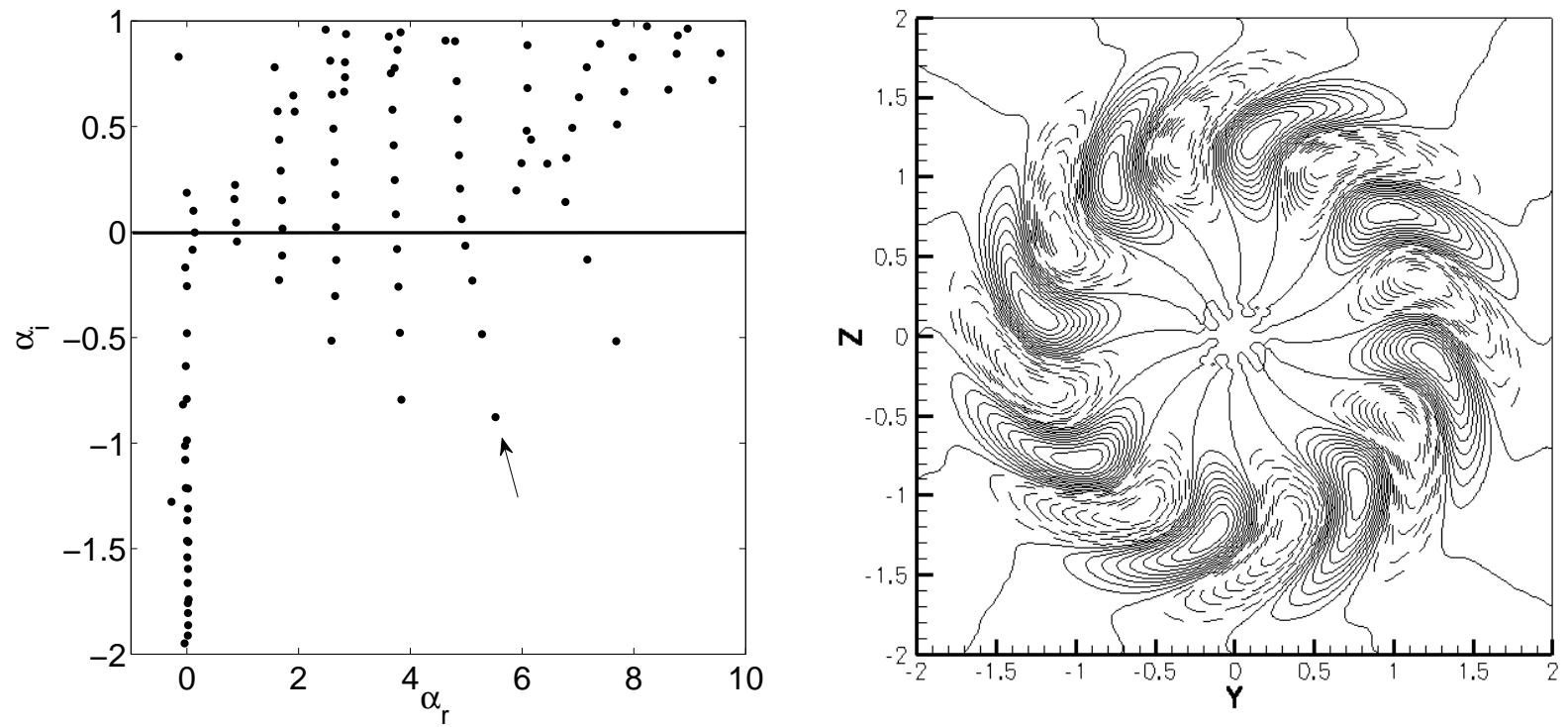

Figure 3. (left) Eigenspectrum of the q-Vortex at $R e=1200, q=0.8$ and $\omega=-2.0$. Arrow points at the most unstable modes of this family that corresponds to a eight lobes $(m=8)$ eigenmode plotted (right) with contours (-0.9:0.1:0.9) of normalized real part of axial velocity amplitude function (dashed lines correspond to negative values).
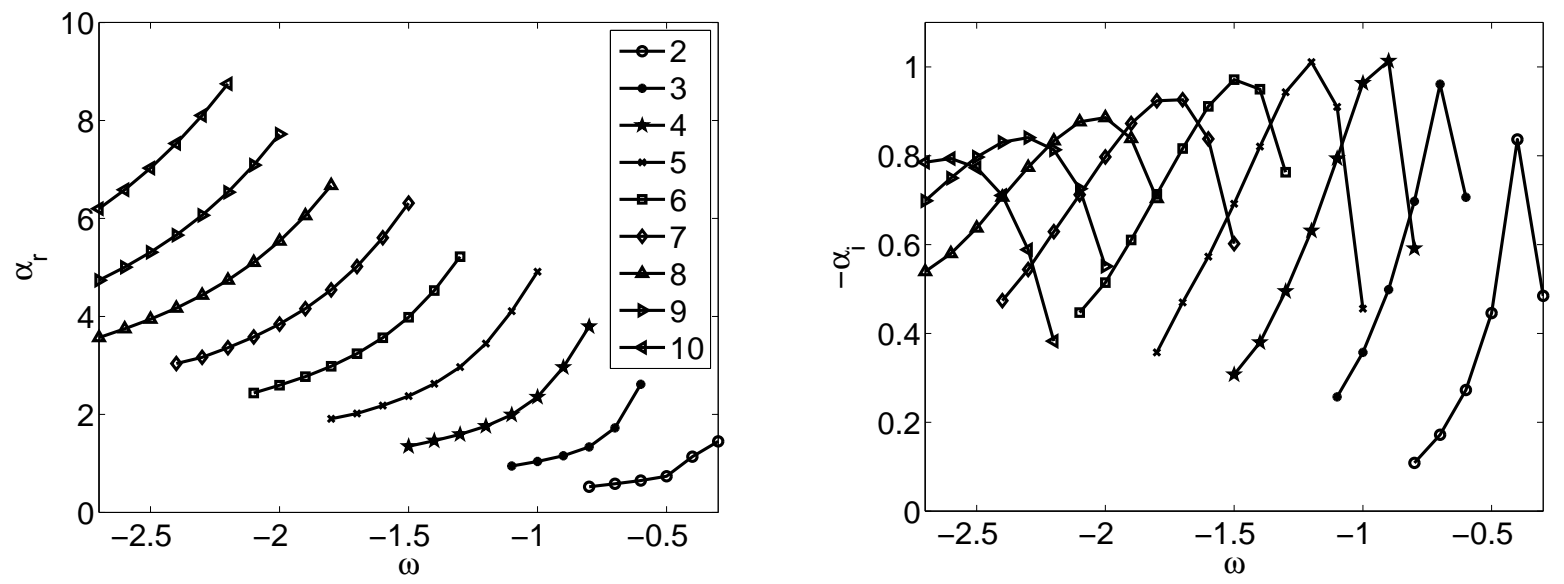

Figure 4. Unstable modes of the q-Vortex flow at $R e=1200, q=0.8$ obtained by temporal BiGlobal analysis. Symbols indicate number of lobes. 

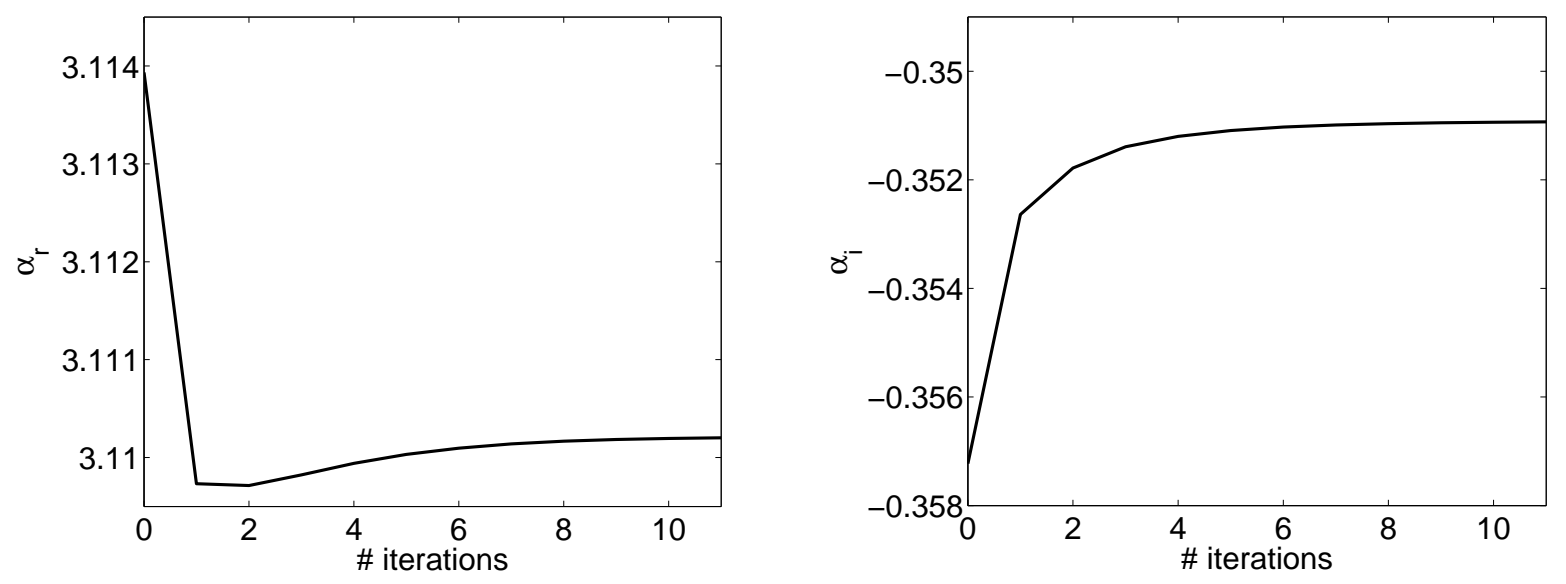

Figure 5. Corrections to the streamwise wavenumber and spatial amplification rate of the initial Spatial BiGlobal solution of the parallel Batchelor vortex flow by including the nonparallel effects by PSE-3D iterations.

the growth rates and eventually stabilizes the modal perturbation.
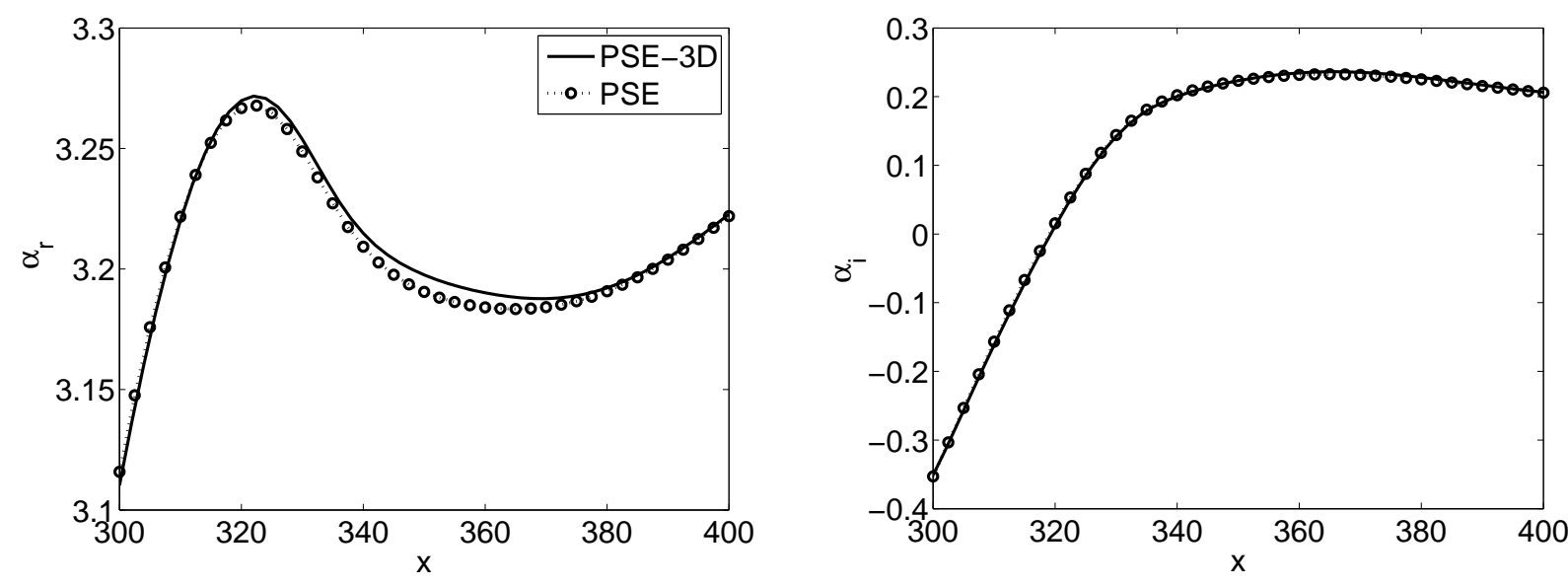

Figure 6. Evolution of the wavenumber (left) and growth rate (right) of the realistic vortex flow for the most unstable mode at initial station $x_{s}=300$ for the selected parameters, comparing PSE and PSE-3D results.

\section{Analysis of a trailing counter-rotating vortex-pair}

As opposed to the isolated vortex considered before, the stability of the non-parallel vortex-pair cannot be analyzed using the standard Parabolized Stability Equations written in cylindrical coordinates, and requires a three-dimensional methodology like the PSE-3D presented in this paper. The three-dimensional flowfield corresponding to a counter-rotating vortex pair is constructed here as the linear sum of two isolated nonparallel vortices (defined by equations 61-63), with vortex cores located at $\left(x_{1}, y_{1}\right)=(2,0)$ and $\left(x_{2}, y_{2}\right)=$ $(-2,0)$. The vortices are defined by $\gamma_{0}=0.9, \delta_{0}=1.0$ and $\kappa=-1.14$. It has been assumed that the radial decay in the velocity components associated with each vortex is fast enough to neglect the interaction between vortices in the basic flow. The computational domain in the $y-z$ plane was truncated at $\left(y_{\text {inf }}, z_{\text {inf }}\right)=(45,40)$, and discretized using $N_{y} \times N_{z}=160 \times 120$ nodes, with mapping parameters $c_{y}=0.92$ and $c_{z}=0.96$. FD-q10 scheme was used for the differentiation in the construction of the linear operators.

Results of the stability analysis corresponding to $R e_{0}=1200$ and $\omega=2$ are shown next. The initial 

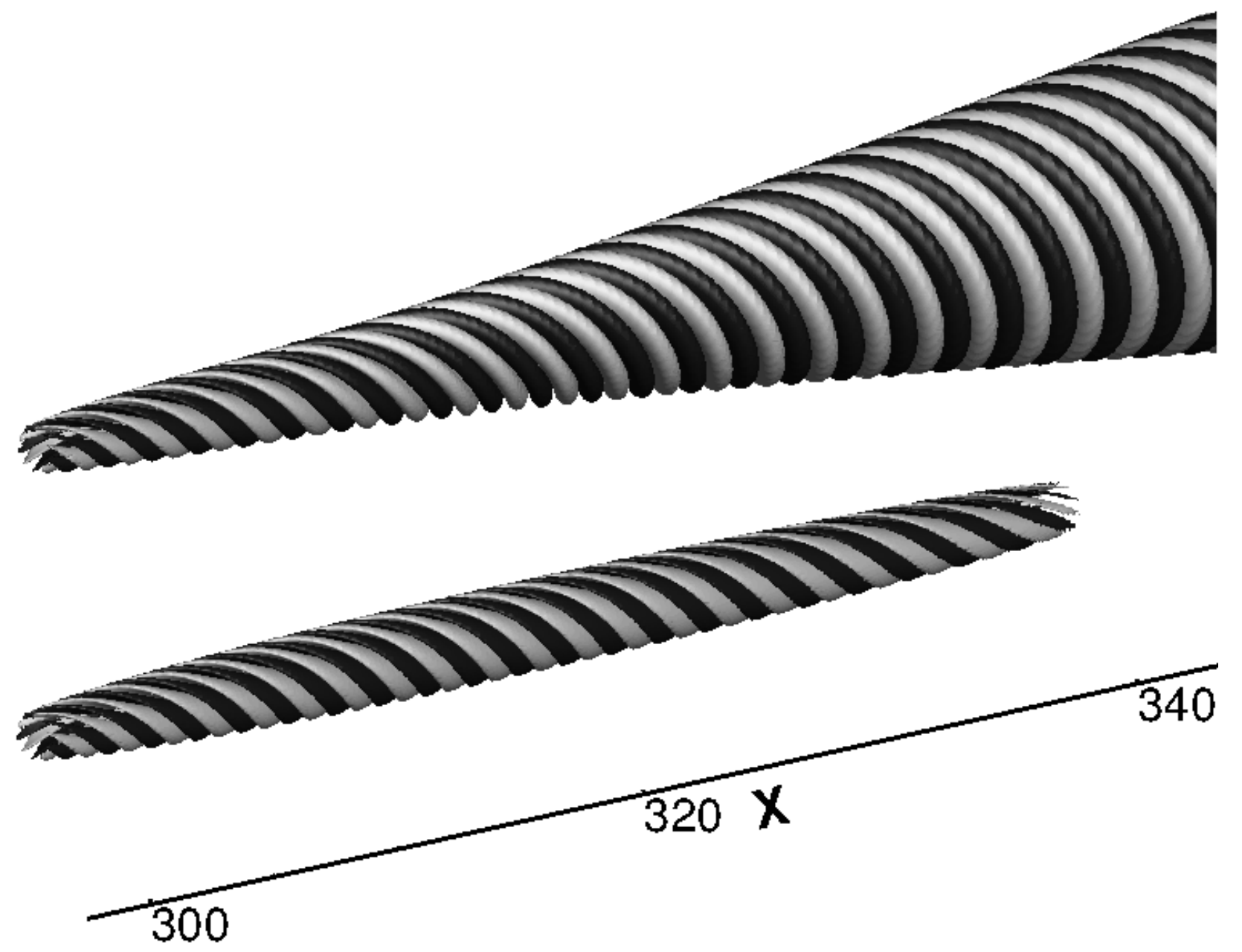

Figure 7. Iso-surfaces $\left(u /\left|u_{0}\right|=1\right.$ and $\left.u /\left|u_{0}\right|=-1\right)$ of axial velocity amplitude function. Upper: BiGlobal analysis of the equivalent parallel vortex with the parameters of the initial position of the analyzed nonparallel vortex (Lower) with PSE-3D. 
position is taken equal to the reference position for which the Reynolds number is defined, $x_{0}=R e_{0} / 4$. Figure 8 shows the real part of the streamwise velocity component corresponding to the leading spatial eigenmode, used as initial condition for the PSE-3D solution. Figure 9 shows the axial evolution of the wavenumber $\alpha_{r}(x)$ and the amplification rate $\alpha_{i}(x)$. Figure (10,upper) shows the iso-surfaces of basic flow axial velocity and Figure (10,lower) shows the evolution of the normalized axial velocity perturbation for the initial most unstable perturbation with the selected parameters.

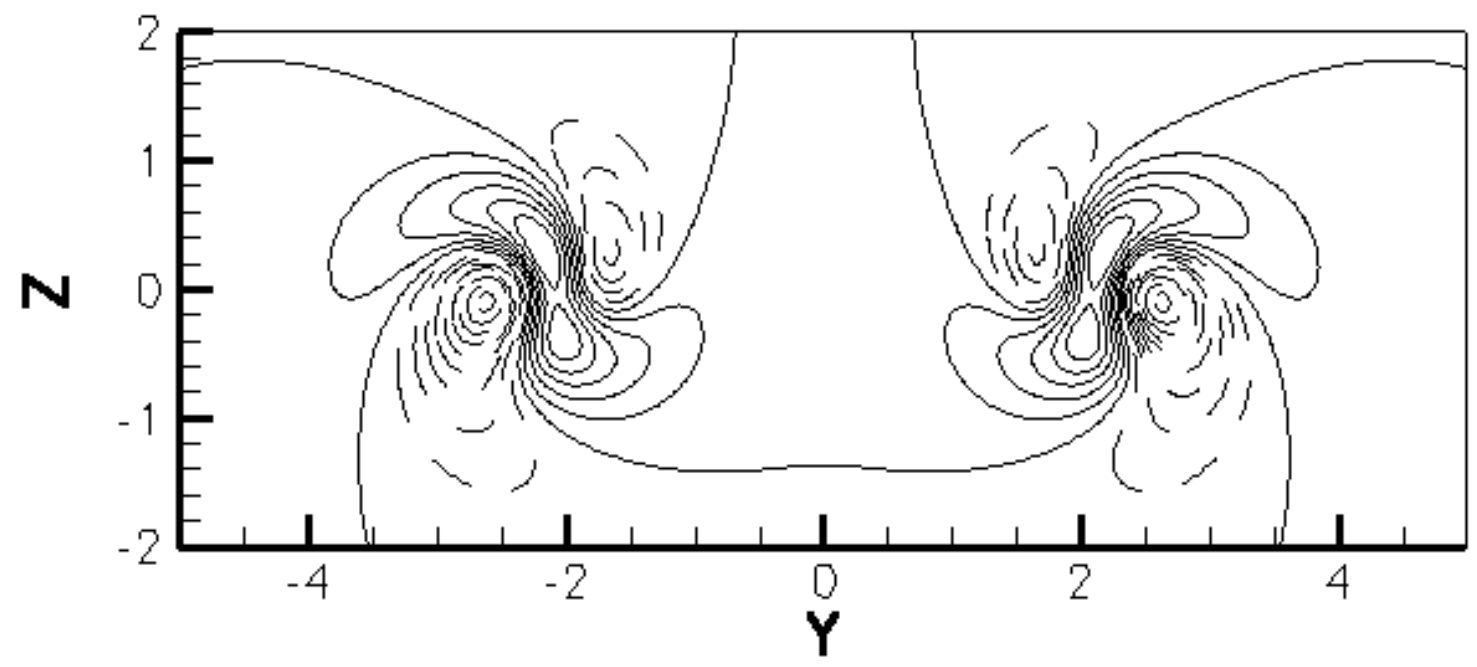

Figure 8. Contours (-0.9:0.1:0.5) of axial velocity amplitude function real part of the most unstable mode for the selected parameters for the realistic vortex flow (dashed lines represent negatives values).
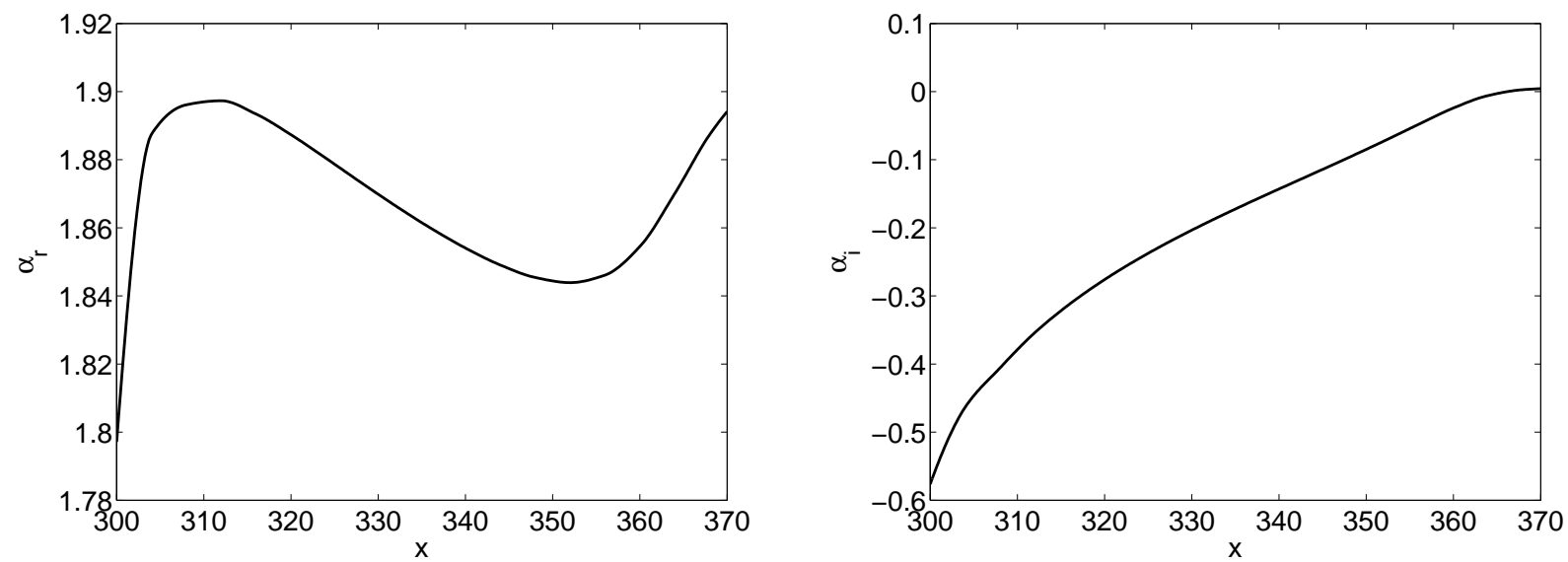

Figure 9. Evolution of the wavenumber (left) and growth rate (right) of the counter-rotating realistic vortexpair flow for the mode shown in Figure 8.

\section{Conclusions}

Spatial BiGlobal eigenvalue problem solutions and PSE-3D analyses were developed and validated with results of Local analysis and PSE. High-order finite-difference schemes were introduced for the discretization of the spatial operator, FD-q, ${ }^{1} \mathrm{SBP},{ }^{38,39} \mathrm{PADE}^{36}$ and $\mathrm{DRP}^{37}$ and results obtained were compared against 


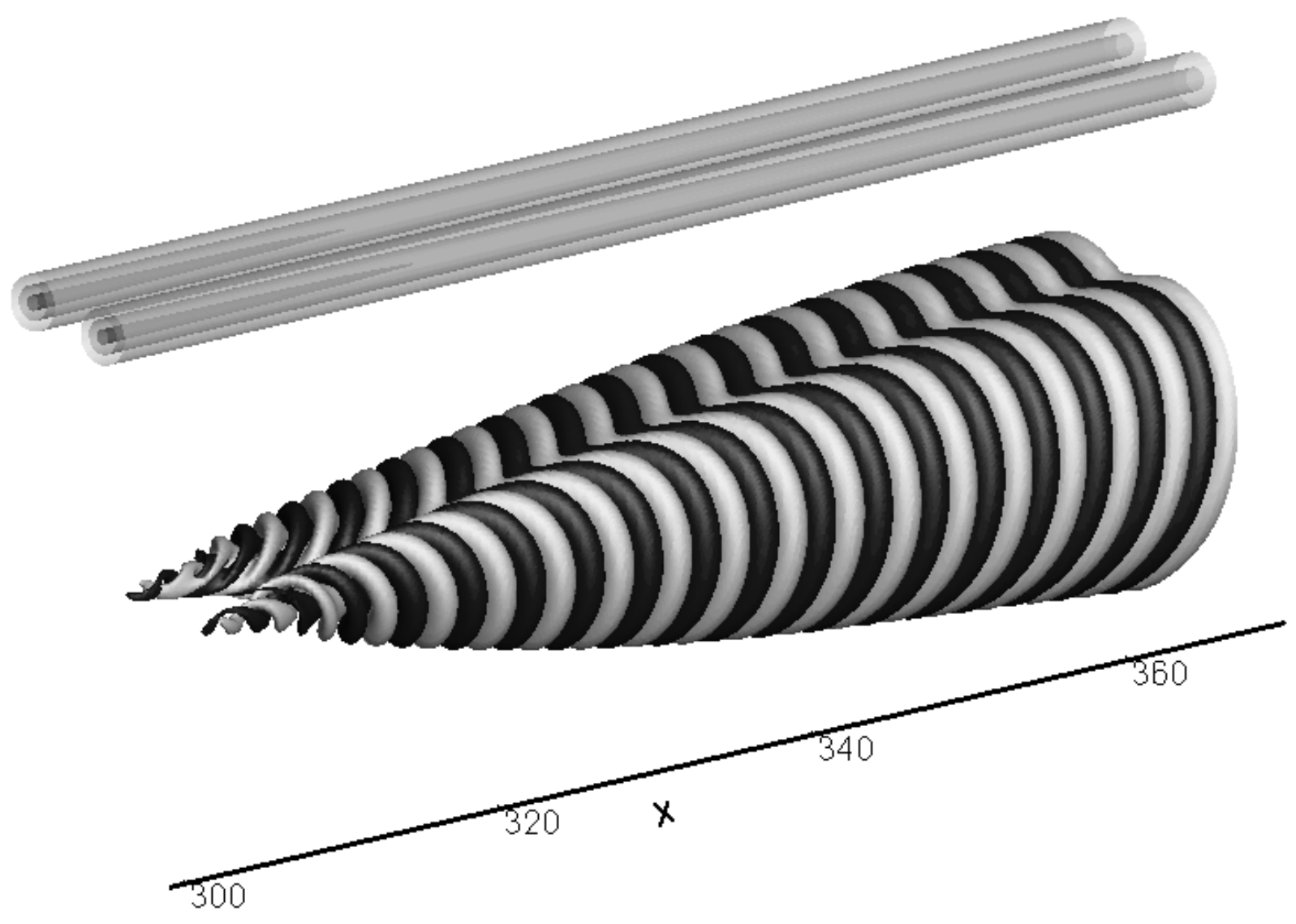

Figure 10. Upper: Basic flow axial velocity and Lower: iso-surfaces $\left(u /\left|u_{0}\right|=100\right.$ and $\left.u / u_{0} \mid=-100\right)$ of axial velocity amplitude function in the range $x \in[300,370]$ 
those delivered by the spectral collocation method based on the Chebyshev-Gauss-Lobbato (CGL) points. The optimal performance shown by the FD-q methods was instrumental in the efficient performance of the PSE-3D analysis. Comparison of spatial BiGlobal and PSE-3D stability analysis of an isolated vortex showed saturation of modal growth due to viscous spread of the model vortices in the downstream direction, as opposed to the uninhibited axial growth predicted by the spatial BiGlobal analysis. The same scenario of axial amplification was also predicted by the PSE-3D methodology in the case of a basic state composed of a pair of viscous, counter-rotating vortices.

\section{Acknowledgments}

Support of the Spanish Ministry of Science and Innovation through Grant MICINN-TRA2009-13648: "Metodologias computacionales para la predicción de inestabilidades globales hidrodinámicas y aeroacústicas de flujos complejos" and discussions with Dr. M. Hermanns are gratefully acknowledged.

\section{References}

${ }^{1}$ Hermanns, M. and J.A., H., "Stable high-order finite-difference methods based on non-uniform grid point distributions," International Journal for Numerical Methods in Fluids, Vol. 56, 2008, pp. 233-255.

${ }^{2}$ Tollmien, W., ber die Entstehung der Turbulenz. 1. Mitteilung, Nachr. Ges. Wiss. Gttingen, Math. Phys. Klasse, 1929.

${ }^{3}$ Drazin, P. G. and Reid, W. H., Hydrodynamic Stability, Cambridge University Press, 1981.

${ }^{4}$ Mack, L. M., "Boundary-Layer Stability Theory," AGARD Report No. 709. Special Course on Stability and Transition of Laminar Flow, 1984, pp. 3-1-3-81.

${ }^{5}$ Schmid, P. and Henningson, D., Stability and transition in shear flows, Springer, 2001.

${ }^{6}$ Herbert, T., "Boundary-Layer Transition - Analysis and Prediction Revisited," AIAA Paper, Vol. 91, 1991, pp. 0737.

${ }^{7}$ Bertolotti, F. P., Herbert, T., and Spalart, P., "Linear and nonlinear stability of the Blasius boundary layer," J. of Fluid Mechanics, Vol. 242, 1992, pp. 441-474.

${ }^{8} \mathrm{Li}, \mathrm{F}$. and Malik, M., "Mathematical nature of parabolized stability equations," R. Kobayashi (Ed.), Laminar-Turbulent Transition, Springer, 1994, pp. 205-212.

${ }^{9}$ Wassermann, P. and Kloker, M., "Transition mechanisms induced by travelling crossflow vortices in a three-dimensional boundary layer," J. Fluid Mech., Vol. 483, 2003, pp. $67-89$.

${ }^{10}$ Chang, C., Malik, M., Erlebacher, G., and Hussaini, M., "Compressible Stability of Growing Boundary Layers Using Parabolized Stability Equations," AIAA Paper, Vol. 91, 1991, pp. 1636.

${ }^{11}$ Herbert, T., "Parabolized stability equations," AGARD Report No. 793. Special Course on Progress in Transition Modelling, 1994, pp. 4(1)-4(34).

${ }^{12} \mathrm{Li}, \mathrm{F}$. and Malik, M., "On the nature of the PSE Approximation," Theoretical and Computational Fluid Dynamics, Vol. 8, 1996, pp. 253-273.

${ }^{13} \mathrm{Li}$, F. and Malik, M., "Spectral analysis of parabolized stability equations," Computers and Fluids, Vol. 26 (3), 1997, pp. 279-297.

${ }^{14}$ Herbert, T., "Parabolized stability equations," Annual Review of Fluid Mechanics, Vol. 29, 1997, pp. 245-283.

${ }^{15}$ Theofilis, V., "Advances in global linear instability of nonparallel and three-dimensional flows," Prog. Aero. Sciences, Vol. 39 (4), 2003, pp. 249-315.

${ }^{16}$ Theofilis, V., "Global linear instability," Annu. Rev. Fluid Mech., Vol. 43, 2011, pp. 319-352.

${ }^{17}$ Broadhurst, M., Theofilis, V., and Sherwin, S., "Spectral element stability analysis of vortical flows," IUTAM Symposium on Laminar-Turbulent Transition, edited by R. Govindarajan, 13-17 December 2004, Bangalore, India, Springer, 2006, pp. 153158.

${ }^{18}$ Broadhurst, M. and Sherwin, S., "The parabolised stability equations for 3D-flows: implementation and numerical stability," Applied Numerical Mathematics, Vol. 58, No. 7, 2008, pp. 1017-1029.

${ }^{19}$ Butler, K. and Farrell, B., "Three-dimensional optimal perturbations in viscous shear flow," Physics of Fluids, Vol. 4(8), 1992.

${ }^{20}$ Heeg, R. and Geurts, J., "Spatial Instabilities of the Incompressible Attachment-Line Flow Using Sparse Matrix JacobiDavidson Techniques," Applied Scientific Research, Vol. 59, 1998, pp. 315-329.

${ }^{21}$ Bridges, T. and Morris, P., "Differential eigenvalue problems in which the parameter appears nonlinearly," Journal of Computational Physics, Vol. 55, 1984, pp. 437-460.

${ }^{22}$ Theofilis, V., "Spatial stability of incompressible attachment-line flow," Theor. Comp. Fluid Dyn., Vol. 7, No. 3, 1995, pp. 159-171.

${ }^{23}$ Khorrami, M., Malik, M., and Ash, R., "Application of spectral collocation techniques to the stability of swirling flows," Journal of Computational Physics, Vol. 81, 1989, pp. 206-229.

${ }^{24}$ Khorrami, M., "On the viscous modes of instability of a trailing line vortex," Journal of Fluid Mechanics, Vol. 225, 1991, pp. 197-212.

${ }^{25}$ Khorrami, M., "Behavior of asymmetric unstable modes of a trailing line vortex near the upper neutral curve," Physics of Fluids A, Vol. 4, 1992, pp. 1310-1313.

${ }^{26}$ Mayer, E. and K.G., P., "Viscous and inviscid instabilities of a trailing vortex," Journal of Fluid Mechanics, Vol. 245, 1992, pp. 94-114. 
${ }^{27}$ Batchelor, G., "Axial flow in trailing line vortices," Journal of Fluid Mechanics, Vol. 4, 1964, pp. 645-658.

${ }^{28}$ Boyd, J., Chebyshev 6 Fourier Spectral Methods, Springer-Verlag, 1989.

${ }^{29}$ Canuto, C., Hussaini, M., Quarteroni, A., and Zang, T., Spectral Methods, Springer, 1988.

${ }^{30}$ Hein, S. and V., T., "On instability characteristics of isolated vortices and models of trailing-vortex systems," Computers E Fluids, Vol. 33, 2004, pp. 741-753.

${ }^{31}$ Saad, Y., "SPARSKIT: a basic tool kit for sparse matrix computations. Version 2." 1994.

${ }^{32} \mathrm{Saad}, \mathrm{Y}$., "Variations of Arnoldi's method for computing eigenelements of large unsymmetric matrices," Linear Algebra Applications, Vol. 34, 1980, pp. 269-295.

${ }^{33}$ Amestoy, P., Duff, I., Koster, J., and L'Excellent, "A fully asynchronous multifrontal solver using distributed dynamic scheduling," SIAM Journal of Matrix Analysis and Applications., Vol. 1, 2001, pp. 15-41.

${ }^{34}$ Amestoy, P., Guermouche, A., L'Excellent, J.-Y., and Pralet, S., "Hybrid scheduling for the parallel solution of linear systems," Parallel Computing, Vol. 2, 2006, pp. 136-156.

${ }^{35}$ Kirchner, N., "Computational aspects of the spectral Galerkin FEM for the Orr-Sommerfeld equation," Int. J. Numer. Meth. Fluids, Vol. 32, 2000, pp. 119-137.

${ }^{36}$ Lele, S., "Compact finite difference schemes with spectral- like resolution," Journal of Computational Physics, Vol. 103, 1992, pp. 16-42.

${ }^{37}$ Tam, C. and Webb, J., "Dispersion-relation-preserving finite difference schemes for computational acoustics," Journal of Computational Physics, Vol. 107, 1993, pp. 262-281.

${ }^{38}$ Strand, B., "Summation by Parts for finite difference approximations for d/dx," Journal of Computational Physics, Vol. 110, 1994, pp. 47-67.

${ }^{39}$ Mattson, K. and Nordström, J., "Summation by parts operators for finite difference approximations of second derivatives," J. Comp. Phys., Vol. 199, No. 2, 2004, pp. 503-540.

${ }^{40}$ Gaster, M., "On the effects of boundary-layer growth on flow stability," J. of Fluid Mechanics, 1974, pp. 66:450-80.

${ }^{41}$ Heaton, C., Nichols, J., and P.J., S., "Global linear stability of the non-parallel Batchelor vortex," Journal of Fluid Mechanics, Vol. 17, 2009, pp. 139-160.

${ }^{42}$ Lessen, M., Singh, P., and Paillet, F., "The stability of a trailing line vortex. Part 1. Inviscid theory," Journal of Fluid Mechanics, Vol. 63, 1974, pp. 753-763.

${ }^{43}$ Lessen, M. and Paillet, F., "The stability of a trailing line vortex. Part 2. Viscous theory," Journal of Fluid Mechanics, Vol. 65, 1974, pp. 769-779.

${ }^{44}$ González, L. M., Theofilis, V., and Meseguer, F., "Galerkin methods for BiGlobal linear instability analysis of vortical flows," Lecture Notes in Computational Science and Engineering., ICOSAHOM 09, 22-26 June, Trondheim, Norway. Springer, 2009. 